\title{
ON CERTAIN WEIGHTED PARTITIONS AND FINITE SEMISIMPLE RINGS
}

\author{
L. B. RICHMOND AND M. V. SUBBARAO
}

ABstract. Let $k$ be a fixed integer $>1$ and define $\tau_{k}(n)=\Sigma_{d^{k} / n} 1$. Thus $\tau_{1}(n)$ is the ordinary divisor function and $\tau_{k}(n)$ is the number of $k$ th powers dividing $n$. We derive the asymptotic behaviour as $n \rightarrow \infty$ of $\boldsymbol{P}_{k}(n)$ defined by

$$
\sum_{n=0}^{\infty} P_{k}(n) x^{n}=\prod_{n=1}^{\infty}\left(1-x^{n}\right)^{-\tau_{k}(n)}
$$

Thus $P_{k}(n)$ is the number of partitions of $n$ where we recognize $\tau_{k}(m)$ different colours of the integer $m$ when it occurs as a summand in a partition. The case $k=2$ is of special interest since the number $f(n)$ of semisimple rings with $n$ elements when $n=q_{1}^{l_{1}} q_{2}^{l_{2}} \ldots$ is given by $f(n)=$ $P_{2}\left(l_{1}\right) P_{2}\left(l_{2}\right) \ldots$

1. Let $k$ be a fixed integer $\geqslant 1$ and define

$$
\tau_{k}(n)=\sum_{d^{k} / n} 1
$$

Thus $\tau_{1}(n)$ is the ordinary divisor function and $\tau_{k}(n)$ is the number of $k$ th powers dividing $n$. We shall derive the asymptotic behaviour of $p_{k}(n)$ defined by

$$
\sum_{n=0}^{\infty} p_{k}(n) x^{n}=\prod_{n=1}^{\infty}\left(1-x^{n}\right)^{-\tau_{k}(n)}
$$

Thus $p_{k}(n)$ is the number of partitions of $n$ where we recognize $\tau_{k}(m)$ different colours of the integer $m$ when it occurs as a summand in a partition. The case $k=2$ is of special interest since the number of semisimple rings with $n$ elements $f_{2}(n)$, when

$$
n=p_{\mathrm{I}}^{\rho_{1}} p_{2}^{\rho_{2}} \ldots
$$

is given by $f_{2}(n)=p_{2}\left(\rho_{1}\right) p_{2}\left(\rho_{2}\right) \ldots$ [1]. Also, when $k$ is large, we expect $p_{k}(n)$ to approach $p(n)$, the number of ordinary partitions.

A generating function for $\tau_{k}(n)$ is given by

$$
\sum_{n=1}^{\infty} \tau_{k}(n) n^{-s}=\zeta(s) \zeta(k s)
$$

LEMMA 1. If $k>1$,

$$
\sum_{n=1}^{N} \tau_{k}(n)=\zeta(k) N+O\left\{N^{1 / k}\right\}
$$

Received by the editors October 24, 1974.

AMS (MOS) subject classifications (1970). Primary 10J20; Secondary 15A17, 10A45. 
and

$$
\sum_{n=1}^{N} \tau_{1}(n)=N \log N+(2 \gamma-1) N+O\left\{N^{1 / 2}\right\}
$$

where $\gamma$ is Euler's constant.

Proof. The case $k=1$ is classical; see, for example, Theorem 320 in [2, p. 264]. The other cases are similar:

$$
\sum_{n<x} \tau_{k}(n)=\sum_{d^{k}<x}\left[x / d^{k}\right]=x \sum_{d^{k}<x} \frac{1}{d^{k}}+O\left\{x^{1 / k}\right\}=x \zeta(k)+O\left\{x^{1 / k}\right\} .
$$

Lemma 1 shows that if we let $F_{\tau}(x)=\Sigma_{n<x} \tau_{k}(n)$, then $F_{\tau}(2 x)=O\left\{F_{\tau}(x)\right\}$ as $x \rightarrow \infty$.

Let us define the function $f_{\tau}$ for real $x>0$ by

$$
f_{\tau}(x)=\sum_{n=1}^{\infty} \tau_{k}(n) e^{-x n}
$$

We define $\alpha$ throughout this paper to be the unique solution of

$$
n=\sum_{m=1}^{\infty} \tau_{k}(m) m\left(e^{\alpha m}-1\right)^{-1} \text {. }
$$

TheOREM 1. Let $m$ be any fixed integer $\geqslant 3$. Let $k \geqslant 1$ be a fixed integer. Then

$$
\begin{aligned}
p_{k}(n)=\left(2 \pi B_{2}\right)^{-1 / 2} \exp \left\{\alpha n-\sum_{n=1}^{\infty} \tau_{k}(n) \log \left(1-e^{-\alpha n}\right)\right\} \\
\times\left[1+\sum_{\rho=1}^{m-2} D_{\rho}+O\left\{f_{\tau}^{1-2 m / 3}(\alpha)\right\}\right] .
\end{aligned}
$$

Here we define $B_{\mu}=B_{\mu}(n)(\mu=2,3, \ldots)$ by

$$
B_{\mu}=\sum_{m=1}^{\infty} \tau_{k}(m) m^{\mu} g_{\mu}\left(e^{\alpha m}\right)\left(e^{\alpha m}-1\right)^{-\mu}
$$

where $g_{\mu}(x)$ is a certain polynomial (the same as in [3] or the $g_{\mu}^{*}$ of Roth and Szekeres [4]) of degree $\mu-1$ and, in particular, $g_{1}(x)=1$ and $g_{2}(x)=x$ so that

$$
B_{2}=\sum_{m=1}^{\infty} \tau_{k}(m) m^{2} e^{d m}\left(e^{d m}-1\right)^{-2}
$$

Finally $D_{\rho}(\rho=1,2, \ldots)$ is defined by

$$
D_{\rho}=B_{2}^{-6 \rho} \sum_{\mu_{1}=2}^{\infty} \cdots \sum_{\mu_{S \rho}=2}^{\infty} d_{\mu_{1}} \cdots \mu_{S_{\rho}} B_{\mu_{1}} B_{\mu_{2}} \cdots B_{\mu_{S \rho}},
$$

the summation being subject to $\mu_{1}+\mu_{2}+\cdots+\mu_{5 \rho}=12 \rho$, and where the $d$ 's are certain numerical constants.

Proof. It is only necessary to note that the conditions of Theorem 1.1 of [3] hold. For convenience we restate the theorem here in terms of the notation of 
the present paper. We say that $\tau_{k}$ is a $P$-function if the integers $l$ such that $\tau_{k}(l) \neq 0$ do not have a common factor $>1$ for all sufficiently large $l$. Then Theorem 1.1 of [3] says:

Let $\tau_{k}(n)$ have properties (I) and (II). Suppose that $\tau_{k}(n)$ is a $P$-function and that $\min _{\tau_{k}(l) \neq 0} \tau_{k}(l)>0$. Suppose furthermore that

$$
\varliminf_{x \rightarrow \infty} \frac{\log F_{\tau}(x)}{\log \log x}>0 .
$$

Let $m$ be any fixed integer $\geqslant 2$. Then

$$
\begin{aligned}
P_{k}(n)= & \left(2 \pi B_{2}\right)^{1 / 2} \exp \left\{\alpha n-\sum_{l=1}^{\infty} \tau_{k}(l) \log \left(1-e^{\alpha l}\right)\right\} \\
& \times\left[1+\sum_{l=1}^{m-2} D_{e}+O\left\{f_{\tau}^{1-2 m / 3}(\alpha)\right\}\right] .
\end{aligned}
$$

It is not necessary to define conditions (I) and (II) since it is shown in [3] that they hold when $F_{\tau}(2 x)=O\left\{F_{\tau}(x)\right\}$ holds, which we have seen does hold. It is clear that $\tau_{k}$ is a $P$-function and, furthermore, $\tau_{k}(l) \geqslant 1$. Also the last condition of Theorem 1.1 holds by Lemma 1. Theorem 1 now follows immediately.

2. In this section we determine the asymptotic behaviour of $p_{k}(n)$ in terms of elementary functions. First of all, from the Mellin inversion formula,

$$
\begin{aligned}
n & =\sum_{m=1}^{\infty} \tau_{k}(m) m\left(e^{\alpha m}-1\right)^{-1}=\sum_{m=1}^{\infty} \sum_{l=1}^{\infty} \tau_{k}(m) m e^{-\alpha m t} \\
& =\frac{1}{2 \pi i} \int_{\sigma-i \infty}^{\sigma+i \infty} \alpha^{-t} \Gamma(t) \zeta(t) \sum_{m=1}^{\infty} \tau_{k}(m) m^{1-t} d t
\end{aligned}
$$

for $\sigma>2,|\arg \alpha|<\pi / 2$.

It is well known that (equation (1.2))

$$
\sum_{m=1}^{\infty} \tau_{k}(m) m^{-t}=\zeta(t) \zeta(t k)
$$

hence,

$$
n=\frac{1}{2 \pi i} \int_{\sigma-i \infty}^{\sigma+i \infty} \alpha^{-t} \Gamma(t) \zeta(t) \zeta(t-1) \zeta((t-1) k) d t .
$$

Lemma 2.1. Let $\alpha$ be defined by equation (1.3) with $k=1$. Then

$$
\alpha=\frac{\pi}{\sqrt{12}} n^{-1 / 2} \log ^{1 / 2} n\left[1+O\left(\frac{\log \log n}{\log n}\right)\right] .
$$

Let $k \geqslant 2$. Then with

$$
b_{k}=\frac{\Gamma(1+1 / k) \zeta(1+1 / k) \zeta(1 / k)}{(\zeta(2) \zeta(k))^{1 / 2+1 / 2 k} 2 k},
$$




$$
\alpha=n^{-1 / 2}(\zeta(2) \zeta(k))^{1 / 2}+n^{1 / 2 k-1} \sqrt{\zeta(2) \zeta(k)} b_{k}+n^{-1} / 8+O\left\{n^{-1 / 2 k-1}\right\} .
$$

Proof. The singularities of $\alpha^{-t} \Gamma(t) \zeta(t) \zeta(t-1) \zeta((t-1) k)$ for $k=$ $1,2, \ldots$ with real part of $t \geqslant 0$ are at $t=0,1,2$ and $1+1 / k$. For $k=1$ there is a double pole, hence the residue at 2 must be evaluated as

$$
\left[(d / d t)\left\{\alpha^{-t} \Gamma(t) \zeta(t)\right\}+2 \alpha^{-t \zeta}(t) \Gamma(t)(\zeta(t-1)-1 /(t-2))\right]_{t=2}
$$

Let us consider the case $k=1$ first. From equation (2.1) and equation (2.2) and the relations

$$
\Gamma^{\prime}(1)=-\gamma, \quad[\zeta(s)-1 /(s-1)]_{s=1}=\gamma, \quad \zeta(\dot{2})=\pi^{2} / 6,
$$

we obtain that

$$
n=\frac{\pi^{2}}{6} \frac{\log (1 / \alpha)}{\alpha^{2}}+O\left\{\alpha^{-2}\right\}
$$

The first part of the lemma follows from this.

For $k=2,3, \ldots$ we obtain from equation (2.1) that

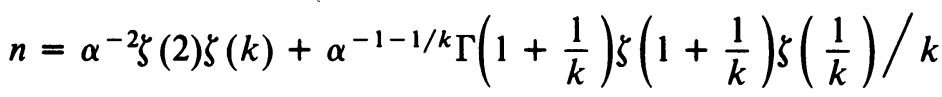

$$
\begin{aligned}
& +\alpha^{-1} \zeta^{2}(0)+O\{1\}
\end{aligned}
$$

and the second part of the lemma follows routinely from this using the fact that $\zeta(0)=-1 / 2$.

LEMMA 2.2. Let $k=1$. Then

$$
\sum_{m=1}^{\infty} \tau_{k}(m) \log \left(1-e^{-\alpha m}\right)=\frac{\pi^{2}}{6 \alpha} \log \frac{1}{\alpha}+O\left\{\alpha^{-1}\right\}
$$

Let $k=2, \ldots ;$ then

$$
\begin{aligned}
-\sum_{m=1}^{\infty} \tau_{k}(m) \log \left(1-e^{-\alpha m}\right) & \\
= & \alpha^{-1} \zeta(k) \zeta(2)+\frac{\zeta(1 / k)}{k} \zeta\left(1+\frac{1}{k}\right) \Gamma\left(\frac{1}{k}\right) \alpha^{-1 / k} \\
& +\frac{1}{4} \log \frac{1}{\alpha}-\frac{(1+k)}{2} \zeta^{\prime}(0)+O\{\alpha\} .
\end{aligned}
$$

Proof. We derive as above that

$$
-\sum_{m=1}^{\infty} \tau_{k}(m) \log \left(1-e^{-\alpha m}\right)=\frac{1}{2 \pi i} \int_{\sigma-i \infty}^{\sigma+i \infty} \alpha^{-t} \Gamma(t) \zeta(1+t) \zeta(t) \zeta(t k) d t,
$$

and then proceed as in the proof of Lemma 2.1. (Note $\zeta^{\prime}(0)=-\frac{1}{2} \log 2 \pi$ and $\Gamma(t)=1 / t-\gamma+\ldots)$

LEMMA 2.3. Let $k=1$. Then

$$
B_{2}=2 \zeta(2) \alpha^{-3} \log (1 / \alpha)+O\left\{\alpha^{-3}\right\} .
$$

Let $k=2,3, \ldots$ Then 


$$
B_{2}=2 \alpha^{-3} \zeta(2) \zeta(k)+O\left\{\alpha^{-2-1 / k}\right\}
$$

Proof. Note that

$$
\begin{aligned}
\sum_{m=1}^{\infty} \tau_{k}(m) m^{2} e^{\alpha m}\left(e^{\alpha m}-1\right)^{-2}=-\frac{d}{d \alpha} \sum_{m=1}^{\infty} \tau_{k}(m)\left(e^{\alpha m}-1\right)^{-1} \\
=\frac{1}{2 \pi i} \int_{\sigma-i \infty}^{\sigma+i \infty} \alpha^{-t-1} \Gamma(t) \zeta(t) t \zeta(t-1) \zeta((t-1) k) d t
\end{aligned}
$$

and the proof proceeds as in Lemmas 2.1 and 2.2.

From Lemmas 2.1, 2.2, 2.3 and Theorem 1, we now obtain, using the facts that $\zeta^{\prime}(0)=-\frac{1}{2} \log 2 \pi$ and $\zeta(2)=\pi^{2} / 6$,

THEOREM 2.1. As $n \rightarrow \infty$,

$$
\log p_{1}(n)=\frac{\pi}{\sqrt{3}} n^{1 / 2} \log ^{1 / 2} n\left[1+O\left\{\frac{(\log \log n)^{2}}{\log n}\right\}\right] .
$$

Let $k=2,3, \ldots$ Then as $n \rightarrow \infty$,

$$
\begin{aligned}
p_{k}(m)= & \exp \left\{2 \pi n^{1 / 2}\left(\frac{\zeta(k)}{6}\right)^{1 / 2}+\frac{\Gamma(1+1 / k) \zeta(1+1 / k) \zeta(1 / k)}{(\zeta(2) \zeta(k))^{1 / 2 k}} n^{1 / 2 k}\right. \\
& \left.-\frac{n^{1 / k-1 / 2}}{4 k^{2}} \frac{\Gamma^{2}(1+1 / k) \zeta^{2}(1+1 / k) \zeta^{2}(1 / k)}{(\zeta(k) \zeta(2))^{1 / 2+1 / k}}+\left(\frac{1+k}{4}\right) \log 2 \pi\right\} \\
& \times \frac{n^{-5 / 8}}{2 \pi^{1 / 4}}\left(\frac{\zeta(k)}{6}\right)^{1 / 8}\left[1+O\left\{n^{-1 / 2 k}\right\}\right] .
\end{aligned}
$$

Note one could obtain as many terms in the asymptotic expansion as required. However, we have not discovered a general formula.

COROLlaRY Let $f_{2}(n)$ denote the number of semisimple rings with $n=p^{m}$ elements. Then with

$$
\begin{gathered}
A=\exp \left(-\frac{9}{4 \pi^{4}} \Gamma^{2}(1.5) \zeta^{2}(1.5) \zeta^{2}(.5)\right) \pi^{3 / 5} 12^{-1 / 4} \log ^{5 / 8} p \\
f_{2}(n) \sim A \log -5 / 8 n \exp \left(\frac{\pi^{2}}{3}\left(\frac{\log n}{\log p}\right)^{1 / 2}\right. \\
\left.+\frac{6^{1 / 2}}{\pi} \Gamma(1.5) \zeta(1.5) \zeta(.5)\left(\frac{\log n}{\log p}\right)^{1 / 4}\right) .
\end{gathered}
$$

Proof. It is only necessary to note that if $n=p^{m}$ then $f_{2}(n)=p_{2}(m)$ (see e.g. (1.1)). 
This corollary provides the asymptotic formula suggested by Knopfmacher on p. 23 of [5]. In [5] it is also shown that $p_{2}(n)$ is the number of nonisomorphic semisimple $n$-dimensional algebras over the Galois field $\operatorname{GF}\left(p^{r}\right), p$ a prime.

This corollary shows that the behaviour of $f_{2}(n)$ is very irregular, since if $n=p$, a prime, then $f_{2}(n)=1$. The average behaviour of $f_{2}(n)$ was originally discussed by Connell [1]. Recently Knopfmacher [5] showed that

$$
\sum_{n<x} f_{2}(n)=\alpha_{1} x+\alpha_{2} x^{1 / 2}+O\left(x^{1 / 3} \log ^{2} x\right)
$$

where

$$
\alpha_{1}=\prod_{r m^{2}>1} \zeta\left(r m^{2}\right)=2.498 \ldots, \quad \alpha_{2}=\zeta\left(\frac{1}{2}\right) \prod_{r m^{2}>1} \zeta\left(\frac{1}{2} r m^{2}\right) .
$$

However, Knopfmacher [6, Theorem E] has shown that for any $\varepsilon>0$ there is an integer $n_{0}(\varepsilon)$ such that

$$
f_{2}(n)<6^{\frac{1}{4}(1+\varepsilon)(\log n) /(\log \log n)} \text { for all } n \geqslant n_{0}(\varepsilon)
$$

while

$$
f_{2}(n)>6^{\frac{1}{4}(1-\varepsilon)(\log n) /(\log \log n)} \text { for infinitely many } n
$$

Moreover,

$$
f_{2}(n)<6^{\frac{1}{4}(1+\varepsilon) \log \log n} \text { for "almost all" } n,
$$

i.e. for all $n$ outside same set of asymptotic denisty zero.

Since any partition of $n$ when a one is added to it gives a partition of $n+1$, it is clear that $p_{k}(n)$ is monotonic increasing. Furthermore, one may derive from Theorem 1, in a manner similar to that of Roth and Szekeres [4], that if $p_{k}^{(l)}(n)$ denotes the $l$ th difference of $p_{k}(n)$ that $p_{k}^{(l)}(n) \sim \alpha^{l} p_{k}(n)$; hence all the differences of $p_{k}(n)$ are positive for $n$ sufficiently large. Below we give a short table of values of $p_{2}(n)$ which are useful for computing $f_{2}(n)$ and the comparison between the asymptotic and true value for certain values of $n$.

$\begin{array}{rrrrrr}n & p_{2}(n) & n & p_{2}(n) & n & p_{2}(n) \\ 1 & 1 & 11 & 79 & 21 & 1549 \\ 2 & 2 & 12 & 115 & 22 & 2025 \\ 3 & 3 & 13 & 154 & 23 & 2600 \\ 4 & 6 & 14 & 213 & 24 & 3377 \\ 5 & 8 & 15 & 284 & 25 & 4306 \\ 6 & 13 & 16 & 391 & 26 & 5523 \\ 7 & 18 & 17 & 514 & 27 & 7000 \\ 8 & 29 & 18 & 690 & 28 & 8922 \\ 9 & 40 & 19 & 900 & 29 & 11235 \\ 10 & 58 & 20 & 1197 & 30 & 14196\end{array}$


$n$

100

200

300

400

500

$$
\text { True Value of } p_{2}(n)
$$$$
2314127129
$$

261229585686401

2469108057914492823

6164394130880718942607

6458643862712466779883980
Asymptotic Value of $p_{2}(n)$

$2.55495 \times 10^{9}$

$2.83594 \times 10^{14}$

$2.65888 \times 10^{18}$

$6.60456 \times 10^{21}$

$6.89497 \times 10^{24}$

We set $\zeta\left(\frac{1}{2}\right)=-1.460, \zeta(1.5)=2.612$ and $\Gamma(1.5)=.8862$ in the asymptotic expression. Since the relative error is $O\left\{n^{1 / 4}\right\}$ we cannot expect a rapid decrease in the relative error. The exact values were computed using the recurrence

$$
n p_{2}(n)=\sum_{k=1}^{n} a(k) p_{2}(n-k) \quad \text { where } a(k)=\sum_{d / k} d \tau_{k}(d) .
$$

This recurrence is obtained by taking the logarithmic derivative of equation (1.0) and comparing coefficients.

\section{REFERENCES}

1. I. G. Connell, $A$ number theory problem concerning finite groups and rings, Canad. Math. Bull. 7 (1964), 23-34. MR 28 \#2149.

2. G. H. Hardy and E. M. Wright, An introduction to the theory of numbers, 4th ed., Oxford Univ. Press, London, 1960. (3rd ed., 1954; MR 16, 673.)

3. L. B. Richmond, A general asymptotic result for partitions, Canad. J. Math. 27 (1975), 1083-1091.

4. K. F. Roth and G. Szekeres, Some asymptotic formulae in the theory of parttions, Quart. J. Math. Oxford Ser. (2) 5 (1954), 244-259. MR 16, 797.

5. J. Knopfmacher, Arithmetical properties of finite rings and algebras, and analytic number theory, J. Reine Angew. Math. 252 (1972), 16-43. MR 47 \# 1769.

6. Arithmetical properties of finite rings and algebras, and analytic number theory. IV, J. Reine Angew. Math. 270 (1974), 97-114. MR 51 \# 389.

Department of Combinatorics and Optimization, University of Waterloo, Waterloo, Ontario, Canada

Department of Mathematics, University of Alberta, Edmonton, Alberta, Canada 